\title{
Paper Extrapolation of Electromagnetic Response from Linear Antennas in Time Domain without Late-Time Instabilities in Numerical Solution of EFI Equation
}

\author{
Anna Witenberg \\ Faculty of Telecommunications, Computer Science and Electrical Engineering \\ UTP University of Science and Technology, Bydgoszcz, Poland
}

https://doi.org/10.26636/jtit.2020.143020

\begin{abstract}
The paper presents a new hybrid method relied upon to solve integral equations of the electric field in time domain and to model linear antennas with pulse excitation. The method consists in a mixed, numerical-analytical description of the process that helps maintain the stability of calculations during the late time phase. As described in the analytical part, the modified spherical Bessel function of the first kind allows for extrapolation with a high degree of accuracy and loworder expressions. The modified spherical Bessel functions of the first kind are of the oscillating character, and their combination with the exponential factor makes them convenient for extrapolation of the answer of the antenna with pulse excitation. New functions are introduced to computational practice.
\end{abstract}

Keywords-electric field integral equation (EFIE), linear antennas, solution of time domain EFIE - late time instabilities.

\section{Introduction}

The problem of obtaining a transient response from an arbitrarily shaped conducting body excited either as an antenna or a scatterer, raises much interest in the electromagnetic research community. Traditionally, in order to determine a wideband response of an antenna or a conducting structure, Maxwell equations are solved either in the time or frequency domain, using one of the many available computational electromagnetic methods.

The method of moments is one of the most popular ones. It allows to perform an electromagnetic analysis in the frequency domain, but in the case of wideband analysis, this approach may be rather complex. Therefore, for wideband analysis, the time domain approach is preferred. Moreover, a time domain formulation includes easier modeling of non-linear and time-varying media.

Using time domain method, wideband information may be obtained by exciting the structure with a narrow pulse
(Gaussian pulse), and by time-stepping the transient response to zero. However, for resonant structures, energy dissipation may be exceedingly slow. So, obtaining the complete response might require thousands of time steps, and the increased probability of numerical dispersion errors needs to be dealt with.

In order to solve the problems referred to above affecting a properly defined integral equation in the time domain, the marching-on in time (MOT) method is usually employed. Unfortunately, this algorithm suffers from a disadvantage consisting in the occurrence of late-time instabilities that take the form of exponentially increasing oscillations whose sign alternates at each time step due to the finite precision of the computation [1]. In order to overcome these instabilities, a specific technique is introduced. It is shown that the time and frequency response caused by a wideband source may be generated based on early-time and low-frequency data, by fitting the response with a summation of orthogonal polynomials (Hermite, Laguerre [2], Bessel-Chebyshev polynomials). But the use of polynomials only in order to represent typical responses (which decay slowly in the time domain) of resonant structures is computationally inefficient and numerically difficult in practice. Furthermore, an extrapolation in either the time or frequency domain is sometimes a numerically unstable process, and its accuracy often cannot be guaranteed [3]-[6].

Therefore, the aim to perform accurate extrapolation in time domain leads to the idea of extend the extrapolation technique by using damped modified spherical Bessel functions of the first kind. Using these functions enable efficiently and accurately modeling the resonance. Thus, the number of required functions is reduced, computation time decreased and the extrapolation accuracy is improved.

In this paper, a technique relying on stable, initial, latetime information is deployed to generate information for the 
entire late time phase. Hence, using a time domain code generates the early-time and initial late-time information. The generation of this information is not only quite simple, but also not very computationally intensive. Next, the new modified spherical Bessel functions of the first kind are used to extrapolate the information in the time domain. In other words, by using early-time and initial late-time data and the extrapolation technique with damped modified spherical Bessel functions of the first kind, stable late-time information is obtained for the remaining portion of the process.

\section{Formulation of Time Domain Electric Field Integral Equation}

Let $S$ denotes a perfectly conducting surface that may be closed or open, with a transient electromagnetic wave incident upon it. This incident wave induces a surface current density $\mathbf{J}(\mathbf{r}, t)$ on $S$, so the induced currents and charges become the sources of a secondary wave. The scattered electric field $\mathbf{E}^{s}(r, t)$ computed from the surface current $\mathbf{J}$ is given by:

$$
\mathbf{E}^{s}(\mathbf{r}, t)=-\frac{\partial \mathbf{A}(\mathbf{r}, t)}{\partial t}-\nabla \Phi(\mathbf{r}, t)
$$

where $\mathbf{A}(\mathbf{r}, t)$ and $\Phi(\mathbf{r}, t)$ are the magnetic vector and the electric scalar potentials, defined as:

$$
\begin{gathered}
\mathbf{A}(\mathbf{r}, t)=\frac{\mu}{4 \pi} \int_{S} \frac{\mathbf{J}\left(\mathbf{r}^{\prime}, \tau\right)}{R} \mathrm{~d} S^{\prime}, \\
\Phi(\mathbf{r}, t)=\frac{1}{4 \pi \varepsilon} \int_{S} \frac{q\left(\mathbf{r}^{\prime}, \tau\right)}{R} \mathrm{~d} S^{\prime} .
\end{gathered}
$$

In addition, the influence of the current density along surface $S$ may be described by the vector potential, and the influence of charge density may be described by the scalar potential.

The permittivity and permeability of the surrounding medium are $\mu\left(\mu=\mu_{r} \mu_{0}\right)$ and $\varepsilon\left(\varepsilon=\varepsilon_{r} \varepsilon_{0}\right)$ respectively. $R=\left|\mathbf{r}-\mathbf{r}^{\prime}\right|$ is the distance between observation point $\mathbf{r}$ and source point $\mathbf{r}^{\prime}$ on $S$, and $\tau=t-R / c$ is the retarded time. $R / c$ is the time for the wave to travel the distance between the observation and the source points. The velocity of propagation in the surrounding medium is $c=(\mu \varepsilon)^{-1 / 2}$.

The surface charge density $q(\mathbf{r}, t)$ is related to the surface divergence of $\mathbf{J}(\mathbf{r}, t)$, based on the equation of continuity:

$$
q(\mathbf{r}, t)=-\int_{0}^{t} \nabla \mathbf{J}\left(\mathbf{r}, t^{\prime}\right) \mathrm{d} t^{\prime} .
$$

Using (4), Eq. (3) may be rewritten in the following form:

$$
\Phi(\mathbf{r}, t)=\frac{1}{4 \pi \varepsilon} \int_{S} \int_{0}^{t} \frac{\nabla^{\prime} \mathbf{J}\left(\mathbf{r}^{\prime}, t^{\prime}\right)}{R} \mathrm{~d} t^{\prime} \mathrm{d} S^{\prime} .
$$

On the surface of the perfectly conducting structure, the electromagnetic boundary condition for the intensity of the electric field requires that:

$$
\left[\mathbf{E}^{i}(\mathbf{r}, t)+\mathbf{E}^{s}(\mathbf{r}, t)\right]_{\tan }=0, \quad \mathbf{r} \in S
$$

The total tangential electric field is zero on the conducting surface for all times, and:

$$
\left[\frac{\partial \mathbf{A}(\mathbf{r}, t)}{\partial t}+\nabla \Phi(\mathbf{r}, t)\right]_{\tan }=\left[\mathbf{E}^{i}(\mathbf{r}, t)\right]_{\tan },
$$

where $\mathbf{E}^{i}(\mathbf{r}, t)$ is the incident electric field (this field is parallel to the surface $S$ ) on the scatterer, and the subscript "tan" denotes the tangential component.

Equations (7), (2) and (5) represent a time domain electric field integral dependence from which the unknown current $\mathbf{J}(\mathbf{r}, t)$ may be determined.

Here, the primary objective is to obtain current distribution on a thin conducting wire (linear antenna), as a function of time when the wire is excited by a narrow electric Gaussian pulse. Furthermore, a "thin wire" approximation is assumed, so the current's components exist along the $z$ axis of the wire only. They are only a function of the $z$ variable and the current flows only on the wire surface. Thus, there is neither current nor conductivity, across the wire. Moreover, the axially directed current may only change its value with distance along the wire, and the wire radius $a$ is much lower than the wavelength and the antenna length.

Under the "thin wire" approximation, we have only the $z$ component for the potentials given by Eqs. (2) and (3), so when the incident electric field impinges on a thin wire, current distribution satisfies the following equation:

$$
\left[\frac{\partial \mathbf{A}(z, t)}{\partial t}+\nabla \Phi(z, t)\right]_{\tan }=\left[\mathbf{E}^{i}(z, t)\right]_{\tan } .
$$

\section{Numerical Solution of TD-EFIE}

The time domain electric field integral equation (TDEFIE) has been solved by applying the marching-on in time (MOT) procedure that is very efficient and simple to code. For the numerical solution of TD-EFIE, the time axis is divided into equal intervals $\Delta t$, and $t_{k}=k \cdot \Delta t, \quad(k=$ $0,1,2, \ldots, \infty)$ is defined. A linear antenna of length $L$ and radius $a$ is divided into $N$ sections with the length of $\Delta z$ each.

The time derivative in Eq. (8) may be approximated by the forward, central or backward finite difference and an explicit or implicit scheme may be obtained [8]. All three cases could be combined (forward, central, backward) into a unified scenario in order to use the same code for implementing the various time - differentiating schemes, by:

$$
\begin{aligned}
\frac{A\left(z, t_{k}\right)-A\left(z, t_{k-1}\right)}{\Delta t} & +(1-p) \cdot \nabla \Phi\left(z, t_{k}\right) \\
& +p \cdot \nabla \Phi\left(z, t_{k-1}\right)=E^{i}\left(z, t_{k-p}\right)
\end{aligned}
$$


where $p=1$ for the forward, $p=0$ for the backward and $p=1 / 2$ for the central finite difference approximation of the derivative for the magnetic vector potential.

Equation (9) is solved numerically in two steps. The first step is connected with the space discretization problem, while the other one concerns the application of time algorithms that use data obtained in the first step. We use the method of moments for the Eq. (8) transformation to a set of the retarded differential equations (the space discretization problem).

For the second step, it is possible to use either the explicit or the implicit algorithm. In the explicit one, the length of time step $\Delta t$ has to satisfy the following Courant condition [9] i.e. $\Delta t \leqslant R_{\min } / c$, where $R_{\min }$ is the smallest distance between the center of the discretization elements and $c$ is the speed of light in the surrounding medium. In the implicit algorithm, the length of the time step may be chosen arbitrarily. Using a larger length of the time step increases the speed of the computation but lowers its accuracy. These approaches are the varieties of the MOT method.

If we focus on time instant $t=t_{k}$, Eq. (9) may be rewritten as [10]:

$$
\begin{array}{r}
2 A\left(z, t_{k}\right)+\Delta t \cdot \nabla \Phi\left(z, t_{k}\right)=2 \Delta t \cdot E^{i}\left(z, t_{k-1 / 2}\right) \\
+2 A\left(z, t_{k-1}\right)-\Delta t \cdot \nabla \Phi\left(z, t_{k-1}\right) .
\end{array}
$$

This is the formula that needs to be solved for the unknown current density $J(z, t)$. It is apparent that the left-hand side of Eq. (10) contains only the unknown currents at time instant $t=t_{k}$, while the right-hand side contains the known currents at $t=t_{k-1}$. Therefore, the algorithm may commence with the assumption that $J\left(t_{0}\right)=0$ to find $J\left(t_{1}\right)$. When current $J\left(t_{1}\right)$ is computed, then it is possible to compute $J\left(t_{2}\right)$, and so on.

\section{Extrapolation Process}

The purpose of extrapolation is to use stable, initial latetime data to determine a representation of the complete response which accurately approximates late-time data even for the very late time phase.

In order to accurately represent the response from the antenna, which is usually slowly decaying over time, hundreds or thousands of orthogonal polynomials are potentially required [2]. The total number of polynomials $N$ constitutes the number of equations to be solved in the extrapolation process. Therefore, it is wise to minimize $N$ while maintaining accuracy. Furthermore, oscillations which may appear in higher-order polynomials may lead to computation errors and numerical instabilities during computations.

Therefore, we chose, as the basis, a new kind of functions, namely - special spherical Bessel functions of the first kind [11]. These functions are incorporated into the summations to accurately and efficiently represent the response.
Let the function $\bar{J}(t)$ denote the approximation of $J(t)$ at $z=0$. In order to determine $\bar{J}(t), J(t)$ is fitted with $N$ functions $j_{n}(t)$ :

$$
J(t) \cong \bar{J}(t)=\mathrm{e}^{-b t} \sum_{n=0}^{N-1} a_{n} j_{n}^{s}(\omega t)
$$

where $b$ is a response damping coefficient, $\omega$ is an angular frequency, $a_{n}$ are the expansion coefficients, and $j_{n}^{s}(\omega t)$ is the $n$-th order modified spherical Bessel functions of the first kind.

Using Eq. (11), accuracy of the extrapolation in the time domain is improved, and the computation time is reduced because fewer terms are needed.

$j_{n}^{s}(\omega t)$ functions may be defined, for $n \geqslant 1$, as:

$$
j_{n}^{s}(\omega t)=(-1)^{n} \cdot \frac{t^{n+1}}{k^{n-1}} \cdot \frac{\partial^{n}}{(t \cdot \Delta t)^{n}}\left(\frac{j_{0}^{s}(\omega t)}{\omega t}\right)
$$

and

$$
j_{0}^{s}(\omega t)=\sin (\omega t), \quad \text { for } \quad n>1
$$

where:

$$
\frac{\partial^{n}}{(t \cdot \Delta t)^{n}}=\underbrace{\frac{1}{t} \cdot \frac{\partial}{\partial t}\left[\frac{1}{t} \cdot \frac{\partial}{\partial t}\left(\frac{1}{t} \cdot \frac{\partial}{\partial t} \cdots\right)\right]}_{n} \text { for } n>1
$$

Alternatively, assuming, that $j_{0}^{s}(\omega t)=\sin (\omega t)$ and

$$
j_{1}^{s}(\omega t)=\frac{\sin (\omega t)}{\omega t}-\cos (\omega t),
$$

a recursive formula may be used:

$$
j_{n+1}^{s}(\omega t)=\frac{2 n+1}{\omega t} \cdot j_{n}^{s}(\omega t)-j_{n-1}^{s}(\omega t),
$$

to generate the $j_{n}^{s}(\omega t)$ functions for $n>1$.

Where $n=2$, we have:

$$
j_{2}^{s}(\omega t)=\frac{3 \sin (\omega t)}{(\omega t)^{2}}-\frac{3 \cos (\omega t)}{\omega t}+\sin (\omega t) .
$$

The new spherical modified Bessel functions of the first type are shown in Fig. 1. With a fixed frequency $\omega$, for large values of the argument, all functions tend to assume the same sinusoidal shape, but have a different phase shift. The major differences between the functions $j_{n}^{1}(\omega t)$ are observed with low argument values (Fig. 1). The difference is the greatest for the first period, and then decreases. It may be said that non-stationary functions whose periods differ initially, tend to become steady.

The functions were designed for the purpose of studying transient phenomena in antennas. However, these functions may effectively model other transient processes that are initially not stationary.

Let $M$ be the number of initial late-time domain samples that are given for $J(t)$. The samples of the original data are $J_{k}=J\left(t_{k}\right)=J(k \cdot \Delta t)$ and a simple set of $N$ unknown weighting coefficients $a_{n}$ is used for time representation. 


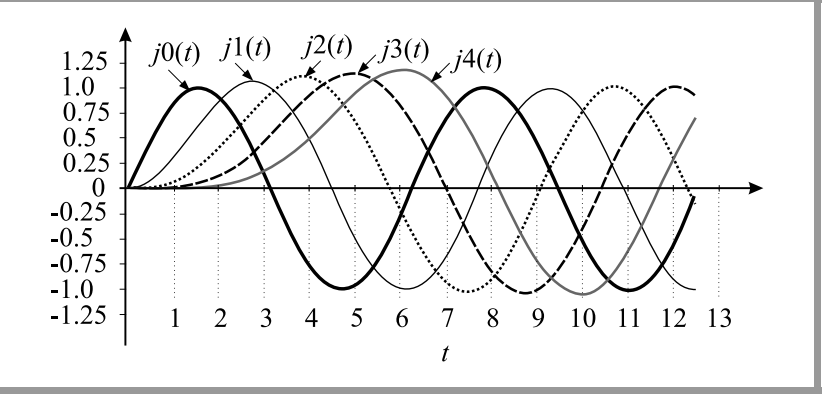

Fig. 1. New modified spherical Bessel functions of the first kind.

These coefficients may be determined by solving a set of linear equations using $M$ samples only.

In a matrix form, the set of equations may be written as:

$$
\left[\Psi_{k j}\right] \cdot\left[a_{n}\right]=\left[J_{k}\right]
$$

where the coefficient matrix components are:

$$
\begin{gathered}
\Psi_{k j}=\sum_{i=0}^{m} j_{k}^{s}\left(\omega t_{i}\right) \cdot j_{j}^{s}\left(\omega t_{i}\right) \cdot \mathrm{e}^{-2 b t_{i}}, \\
J_{k}=\sum_{i=0}^{m} j_{k}^{s}\left(\omega t_{i}\right) \cdot \mathrm{e}^{-b t_{i}} \cdot J\left(t_{i}\right) .
\end{gathered}
$$

Matrix $\left[\Psi_{k j}\right]$ is symmetric and positively definite, assuring a single-valued solution of the matrix from Eq. (18).

The least squares solution for $a_{n}$ may be found. By solving Eq. (18), we may obtain the value of the coefficient vector $\left[a_{0}, a_{1}, \ldots, a_{n}\right]^{T}$. The reconstruction of signal $\bar{J}(t)$ in latetime may be performed by multiplying the coefficient vector with the respective $j_{n}^{s}(k t)$ functions. If the extrapolation is successful, $\bar{J}(t)$ closely approximates $J(t)$, including in the late-time range.

\section{Numerical Example}

In this section, we present an example in order to verify the technique presented in the previous section. Software has been relied upon to evaluate current distribution on a linear antenna using the time domain electric field integral equation. The antenna is assumed to be perfectly conducting and it was excited with a plane wave with a Gaussian profile in time. The excitation has the following form:

$$
E^{i}(t)=\frac{4 E_{0}}{c T \sqrt{\pi}} \mathrm{e}^{-\left[\frac{4}{T}\left(t-t_{0}\right)\right]^{2}},
$$

where $E_{0}$ is the amplitude of the incoming wave, $t_{0}$ is a delay and $T$ controls the pulse width.

In the presented example, we assumed that: $E_{0}=$ $120 \pi \mathrm{V} / \mathrm{m}, t_{0}=8 \mathrm{~ns}, T=6 \mathrm{~ns}$. The length of the antenna $L=1 \mathrm{~m}$, the radius of the antenna $a=0.002 \mathrm{~m}$ and the number of segments is chosen to be $N_{s}=20$. The remaining parameters of Eq. (11) are obtained from the MOT algorithm: damping coefficient $9 b=0.067714$ and angular frequency $\omega=0.89264$.
The extrapolated time domain response is compared with the time domain response obtained with the use of MOT software. The time step used in MOT is:

$$
\begin{gathered}
\Delta t_{1}=\frac{\Delta z}{c}=0.1667 \mathrm{~ns}, \\
\Delta t_{2}=2 \cdot \frac{\Delta z}{c}=0.3334 \mathrm{~ns}, \\
\Delta t_{3}=2.25 \cdot \frac{\Delta z}{c}=0.3751 \mathrm{~ns},
\end{gathered}
$$

where $\Delta z$ denotes the length of the segment and $\Delta z=L / N_{S}$. Moreover, the time domain responses calculated by using MOT are presented in the Figs. 2, 3 and 4.

One may notice that for $\Delta t>\Delta z / c$ the time domain response contains late-time instabilities.

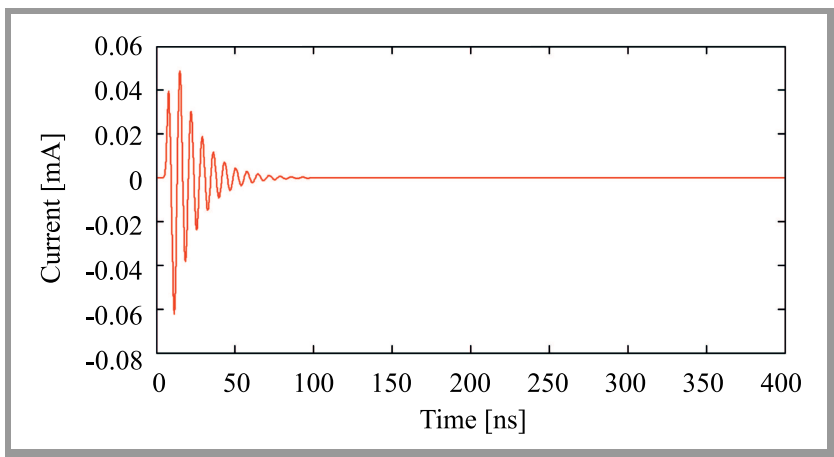

Fig. 2. Time domain response calculated by using MOT and $\Delta t_{1}=0.1667 \mathrm{~ns}$.

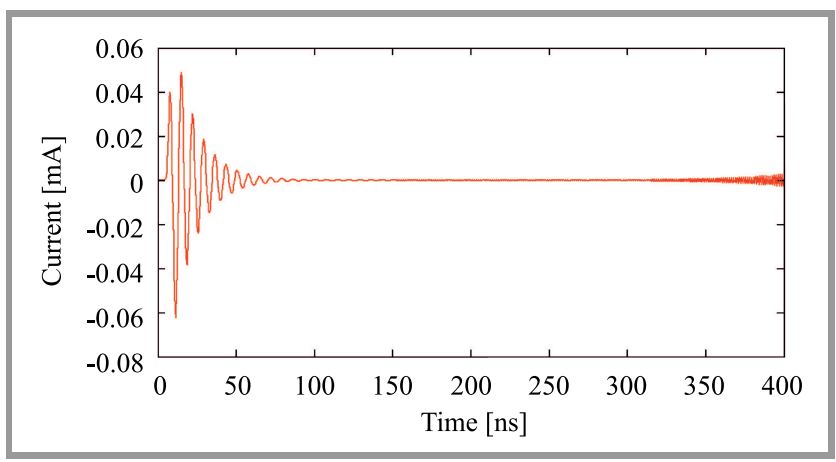

Fig. 3. Time domain response calculated by using MOT and $\Delta t_{2}=0.3334 \mathrm{~ns}$.

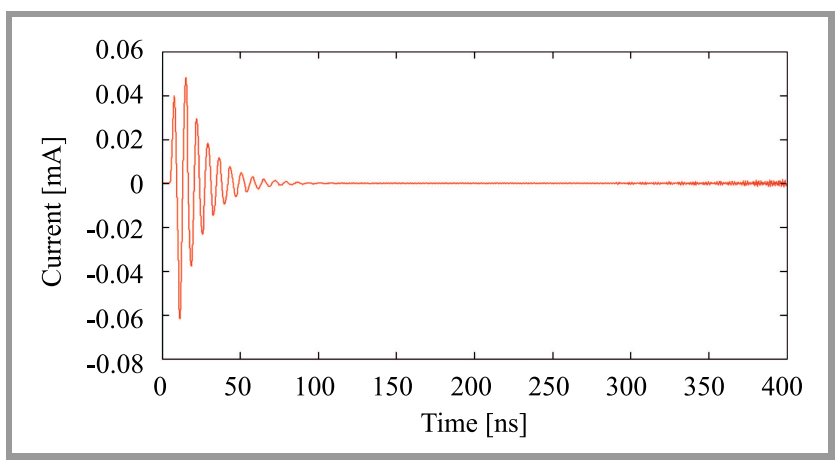

Fig. 4. Time domain response calculated by using MOT and $\Delta t_{3}=0.3751 \mathrm{~ns}$. 
Using the MOT algorithm, the time domain data are obtained from $t=8 \mathrm{~ns}$ to $112 \mathrm{~ns}$. In this time interval, the time domain responses from the MOT algorithm are identical for time steps $\Delta t>\Delta z / c$.

Assuming that only 8 time-data points from $t=98.669 \mathrm{~ns}$ to $t=111.06 \mathrm{~ns}$ with $\Delta t=1.77 \mathrm{~ns}$ are available and that the order of the polynomial is chosen to be two, only three weighting coefficients $a_{n}\left(a_{0}, a_{1}\right.$ and $\left.a_{2}\right)$ are needed.

By solving Eq. (18) using the available data, and then using coefficients $a_{0}, a_{1}$ and $a_{2}$, the time domain response is extrapolated over the late-time of $t=98.669 \mathrm{~ns}$ to $t=400 \mathrm{~ns}$. The approximation is also effective for a period over $t=400 \mathrm{~ns}$.

The result of the computation is:

$$
\begin{aligned}
& \bar{J}(t)=e^{-0.067714 \cdot t} \cdot\left[-0.244 \cdot \frac{\sin (\omega t)}{t^{2}}+\right. \\
& +0.0226 \cdot \frac{\sin (\omega t)}{t}+0.2175 \cdot \frac{\cos (\omega t)}{t}+ \\
& +0.144 \cdot \sin (\omega t)-0.02014 \cdot \cos (\omega t)]
\end{aligned}
$$

for $t \in\langle 98.669, \infty)$ and $\omega=0.89264$.

Using Eq. (22), for the time from $t=0$ to $98.669 \mathrm{~ns}$, the stable current waveform is calculated numerically by the MOT method, and for times greater than $t=98.669 \mathrm{~ns}-$ the current waveform is described by Eq. (22). As long as the numerical solution is stable, the numerical solution is used, then approximation by Eq. (22) is performed. In this way, stability is obtained throughout the process considered. Stability of the approximation is illustrated in Fig. 5.

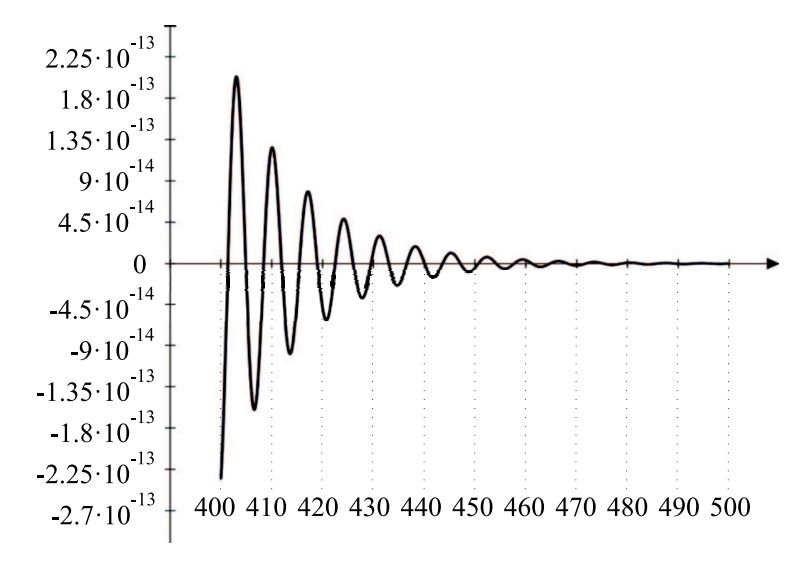

Fig. 5. Illustration of the absolute stability of the Eq. (18).

The extrapolated time domain response is agreeable with the MOT data. The results are satisfactory, as they compare well with the actual solution. The relative errors of the approximation are in the range from $0.0012 \%$ to $0.0637 \%$. In this way, by using early-time data, we obtained stable late-time information.

\section{Conclusions}

In this paper, a technique using stable initial late-time information is relied upon to generate stable information for the entire late-time phase.

The proposed solution offers a considerable advantage in the form of its ability to determine approximating functions of small orders, which approximate and extrapolate data obtained from the stable antenna response with a high degree of accuracy.

For a simple but effective approximation, new special spherical Bessel functions have been introduced. Although the purpose of constructing these functions was to study transient states in antennas, they may be useful for describing other initially non-stationary processes.

\section{References}

[1] P. D. Smitd, "Instabilities in time marching methods for scattering: cause and rectification", Electromagnetics, vol. 10, no. 4, pp. 439-451, 1990 (DOI: 10.1080/02726349008908256).

[2] Y. S. Chung, T. Sarkar, B. Ho Jung, and M. S. Palma“Solution of time domain electric field integral equation using the Laguerre polynomials", IEEE Trans. on Antenn. and Propag., vol. 52, no. 9, pp. 2319-2328, 2004 (DOI: 10.1109/TAP.2004.835248).

[3] A. D. Vechinski and S. M. Rao, "A stable procedure to calculate the transient scattering by conducting surfaces of arbitrary shapes", IEEE Trans. on Antenn. and Propag., vol. 40, no. 6, pp. 661-665, 1992 (DOI: 10.1109/8.144600).

[4] A. Sadigh and E. Arvas, "Treating the instabilities in marching-onin-time method from a different perspective", IEEE Trans. on Antenn. and Propag., vol. 41, no. 12, pp. 1695-1702, 1993 (DOI: 10.1109/8.273314).

[5] X. Wang, R. A. Wildman, D. S. Weile, and P. A. Monk, "A finite difference delay modeling approach into the discretization of the time domain integral equations of electromagnetics", IEEE Trans. on Antenn. and Propag., vol. 56, no. 8, pp. 2442-2452, 2008 (DOI: 10.1109/TAP.2008.926753).

[6] P. J. Davis, "On the stability of time-marching schemes for the general surface electric field integral equation", IEEE Trans. on Antenn. and Propag., vol. 44, no. 11, pp. 1467-1473, 1996 (DOI: 10.1109/8.542071).

[7] S. M. Rao, Time Domain Electromagnetics. London: Academic Press, 1999 (ISBN: 9780125801904).

[8] S. D. Weile, G. Pisharody, N. Y. Chen, B. Shanker, and E. Michielssen, "A novel scheme for the solution on the time-domain integral equations of electromagnetics", IEEE Trans. on Antenn. and Propag., vol. 52, no. 1, pp. 283-295, 2004 (DOI: 10.1109/TAP.2003.822450).

[9] G. Manara, A. Monorchio, and R. Reggiannini "A space-time discretization criterion for a stable time-marching solution of the electric field integral equation", IEEE Trans. on Antenn. and Propag., vol. 45, no. 3, pp. 527-532, 1997 (DOI: 10.1109/8.558668).

[10] B. H. Jung and T. H. Sarkar, "Time-domain electric-field integral equation with central finite difference", Microw. and Optic. Technol. Lett., vol. 31, no. 6, pp. 429-434, 2001 (DOI: 10.1002/mop.10055).

[11] A. Witenberg, M. Walkowiak, and J. Małecki, "A new analytical and numerical method for describing the response of a linear antenna for pulse excitation submission", in Proc. IIEEE Int. Conf. on Microw., Antenn., Commun. and Electron. Syst. COMCA 2019, Tel Aviv, Israel, 2019 (DOI: 10.1109/COMCAS44984.2019.8958452). 


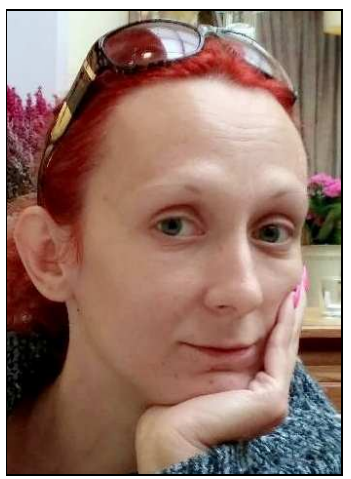

Anna Witenberg received her M.Sc. degree in Digital Telecommunications Engineering from the Faculty of Electronics and Computer Science of the Koszalin University of Technology, Koszalin, Poland, in 2006, and a Ph.D. degree in Technical Sciences (Telecommunications) from the Faculty of Telecommunications, Computer Science and Electrical Engineering of the University of Technology and Life Sciences, Bydgoszcz, Poland, in 2015. Since 2014, she has been working at the Faculty of Telecommunications, Computer Science and Electrical Engineering at the University of Technol- ogy and Life Sciences in Bydgoszcz. Her interests include telecommunications theory, radio communication systems, antennas, medical applications of electromagnetic field and human-electromagnetic field interactions. As an author or co-author, she wrote approximately 60 scientific papers, including those published in international magazines and in papers from reputable conferences devoted to aerials and to the protection of critical infrastructures.

(iD) https://orcid.org/0000-0001-5512-894X

E-mail: anna.witenberg@utp.edu.pl

Faculty of Telecommunications, Computer Science and Electrical Engineering

UTP University of Science and Technology

Al. prof. S. Kaliskiego 7

85-796 Bydgoszcz, Poland 